\title{
AH ATTEMPT TO AUTOMATIC THESAURUS CONSTRUCTION FROY AN ORDINARY JAPANESE LANGUAGE DICTIONARY
}

Hiroaki Tsurumaru

Department of Electronics

Nagasaki University

Nagasaki 852, JAPAN

\section{ABSTRACl'}

How to obtain hierarchical relations (e.g. superordinate -hyponym relation, synonym relation) is one of the most. important problems for thesaurus construction. A pilot system for extracting these relations automatically from an ordinary Japanese language dictionary (Shinmeikai Kokugojiten, published by Sansei-do, in machine readable form) is given. The features of the definition sentences in the dictionary, the mechanical extraction of the hierarchical relations and the estimation of the results are discussed.

\section{INIRODUCTTON}

A practical sized semantic dictionary (thesaurus as wide sence) is necessary for advanced natural language processing. We have been studying how to obtain semantic information for such semantic dictionary from a Japanese language dictionary (Shinmeikai Kokugojiten, published by Sanseido, in machine readable form)(1) containing about 60,000 entries.

A dictionary contains meanings and usages of practical size of general words. Especially, definition sentences LDS: a brief notation are important sources of information for meanings of general words. Generally, DS of an entry word [EW: a brief notation\} is defined by qualifying its superordinate word or synonyms or hyponyms. We call these words definition words \{DH:a brief notation\}.

He have been developing a system for extracting autumaticaliy DW related to EW from its DS, and for deciding the DW-EW relation $\left(f_{i}\right)$. By this system, (hierarchical) relations among entry words in the dictionary are to be established.

We constructed a sub-system for extracting DSs corresponding to parts of speech, infrected forw and meaning (definition) number of each entry word( 7 ).

In this paper, the features of DSs in the Japanese dictionary, an outline of the pilot system and the results of experiment will be discussed.

\section{FEATURES OF DS IN ORDINARY JAPANESE LANGUAGE DICTIOHARY}

\subsection{STRUCTURE OF DS}

The typical examples of DSs are as follows:

(1)【折尺(zigzag rule)】：ㄴしまっておける(possible to be folded) 20 L(rule)。

(2)【山路(mountain path)】：「...O中の(in)小道(narrow path)

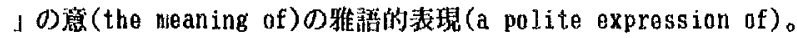

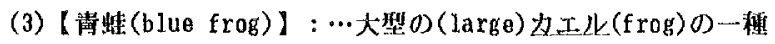

Toru Hitaka Sho Yoshida

Department of Electronics

Kyushu University 36

Fukuoka 812, JAPAK

(a kind of)。

(4)【擦御 (respectful daughter-in-10w)】: 蝊(daughter-inlok)に対する(for) 繁敬語(a respectful word)。

(5)【秋虫 (autumn insect)】: ZZZ出 (boll-ing insect). アツムシ (a kind of crickel)など(etc)。

Where the brackets $((\cdots))$, underline, and parentheses $((\cdots))$ denote EW, DW, and an English translation for the preceding Japanese phrase respectively.

In (1), the final word is $\mathrm{DH}$ and superordinate-hyponym relation(DW $>$ EW) holds between the DW and the EW.

In (2), DH is the final word in hook brackets $(\ulcorner\cdots \downarrow)$ and DW $>$ EW holds. The expression “ $\Gamma \ldots .$. O意O雅語的表堄” is called a functional expression \{FE:a brief notation\}. The (compound) word "雅語的表理" in the $\mathrm{FE}$ is called a functional word \{FH: a brief notation\}. In this case, the FW denotes a usage of the Fi.

In (3), DW is just before the FE " $O$ - $\rightarrow$ 種" and $D W>E W$ holds. In this case, the l'E prescribes the DW $>$ EW explicitly. The ward "--茂" is the FW.

In (4), DW is just before the FE “に対す当然牧語” and the synonyrous relation(DHEEW) holds between the $D H$ and the EH. The IW "放語” denotes a usage of the EW.

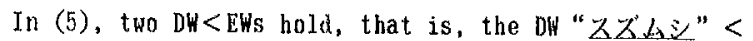

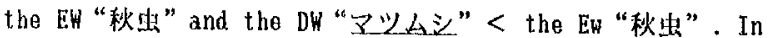
this case, the number of DHs are more than one, DW isn't modified and the FE is the word “なと”. The FW is identiCal with the FE. (Notes: “など" is a sub-postpositive signifying exemplification.)

The features of DSs in the dictionary are as follows:

(a) Generaly, the final word in DS is DH.

(b) In some cases, the final expression in $\mathrm{DS}$ is $\mathrm{FE}$ assigning semantic relation between DW and $E W$, and $D H$ is just before the $F E$.

(c) Genraly, DW is modified by another phrase(modifier).

(d) In sofie cases, DS contains more than one DH.

The following general structure is obtained according to these features.

$\cdots([M O D I F I E R] \cdot D W)$ B $[$ [FE]

Notes) $[\cdots]$ : optional constituent (..) : reguired constituent

*: sequence of coordinate constituent(e.g. ·, と)

- : concatination sywbol which is diferent frow coordinate constituent $(\cdot)$

For convenience of explanation, the general structure is divided into the following two types.

(I) TYPE I : $\cdots($ ([HODIF]ER] $\cdot \mathrm{DH}) *$ 。

(II) IYPE II : $\cdots($ (MODIF]ER] $\cdot \mathrm{DH}) * \cdot \mathrm{FE}_{0}$ 
In TYPE I, the final word is DW. In TYPE II, the final expression is FE, and DW is just before the FE.

\subsection{DW-EH RELATION IN DS}

We will propose the following assumptions according to above-mentioned features in order to extruct the DH-EW relations frow DSs of the general structure.

(1) When DS is in TYPE I, DS $\equiv$ EY. Because DS is a phrase (or a compound word) as wide sence.

(2) When DS is in TYPEII, SS P FE EW.

Where $\rho$ FE is binary relation assigned by FE, and SS

is the shortened DS corresponding to ([MODIFIER] . DW)*.

(3) [MODIFIER] $\cdot \mathrm{W} \leqq \mathrm{W}$

(4) ([MODIFIER $\left.\left.{ }_{i}\right] \cdot W_{i}\right) * \geq[$ [MODIFIER $]$

Where $i, j=1 \sim n, \quad W$ is arbitrary word.

The following general algorithm for deciding the DW-EW relations is obtained by means of these assumptions.

(I) DS is in TYPE I (DS dosn't include FE),

(A) DW is modified,

$(\alpha)$ The number of DW is only one, then $D H>E W$

$(\beta)$ The number of DW are wore than one, then $C D$

(B) DH isn't modified,

$(\alpha)$ The number of DW is only one, then $D W \equiv E W$

$(\beta)$ The number of DW are more than one, then $D W<E W$ (II) DS is in TYPE II (DS includes FE),

(A) $D H$ is modified,

$(\alpha)$ The number of $D W$ is only one, OFE is ' $>$ ' or ' $\equiv$ ', then DW $>$ EW otherwise CD.

$(B)$ The number of DW are more than one, then CD

(B) DW isn't modified,

$(\alpha)$ The number of DW is only one, then DW $\rho$ FE EW

$(\beta)$ The number of $D W$ are wore than one, $\rho F E$ is ' $<$ ', then $D H<E H$ otherwise $C D$.

CD denotes that DH-EW relation isn't extracted mechanically from DS. In this case, the extraction of DW-EW relation needs human support at this stage.

2.3 FEATURES OF FE

FE prescribes hierarchical relations(e.g. DH>EW, DH<EW, $D H=E H$, or $D W \equiv E W$ ) or whole-part relation(DW》EW). (e.g. On

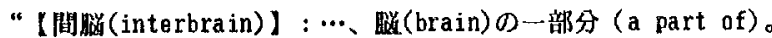
", the FE “の一部分” prescribes DW》EW explicitly.)

Besides these relations, another relation between $D W$ and EW are prescribed by special FEs(e.g. "O下(under)"), which is called associative relation(R).

There are so many FEs that they are mainly divided into four patterns called functional patterns \{FP: a brief notation\}. FP is expressed by means of regular expression. FP is necessary for extracting $F E$ and DW-EW relation information(i.e. information neccessary for deciding the DH-EW relations) assigned by the FE. FP also designates a place of $\mathrm{DW}$ in DS.

Hain features of FP are as follows:

(1) Type100: $\left[\ldots D_{\lrcorner} \cdot \sigma^{*} \cdot \mathrm{FW}\right.$

(2) Type200: $\cdots \mathrm{DW} \cdot(の \cdot \mathrm{FW}) *$

(3) Type $300: \cdots D W \cdot P \cdot F W$

(4) Type $400: \cdots D$ •など
Notes) $\sigma^{*}$ is arbitrary character string,

$(\cdots) *$ is repitation of $(\cdots)$,

$P$ is special phrase(e.8. に奶する),

- is concatination symbol.

He got about one hundred seventy FWs. These are classified into two groups. In one group (contained 64 FWs), the FWs contain explicitly DW-EW relation inforwation. In the other group (contained $105 \mathrm{FWs}$ ), sowe of the FWs contain usages of the EWs, which are also important to thesaurus.

We have constructed a FW dictionary which includes FP and DW-EW relation information corresponding to the FP.

\section{SYSTEM FOR EXTRACTING DH-EW (HIERARCHICAL) BELATION}

The system consists of the following four steps. (1) Extraction of EW and DS

(a) Extraction of EH, its DS, the part of speech of the EW, the definition number of the DS from the dictionary.

(b) Transformation of the extracted DS to the ordinary Japanese sentence's form(called the normalized DS). Because several contents (meanings) are thrown into one DS by means of parentheses or dot ' ' in the dictionary.

(2) Extraction of FE and DH-EH relation information

The FW Dictionary is used.

(a) When DS dosen't include FH, DS is in TYPE I.

(b) When DS includes FW and conforms FP, DS is in TYPE II.

(c) When DS includes FW but doesn't conform FP or when DS includes more than one FW, the DS is picked out as check data.Because it is difficult to distinguish between DW and FW or to extract DW-EW relation information mechanically.

(3) Extraction of DW and DW-EW relation information

A general word dictionary (containing about 75,000 noun words)(5) is used, in which the character strings of entry words were arranged in inverse order (from right to left). DWs are basically extracted by means of longest matching method, because there is ordinarily no space between two adjacent words in the Japanese sentence. In addition to this, there are the following problems.

(a) The 'hiragana' notation is of ten used(e.g. 屯0き [物差し]).

(b) The names of animals and plants are described by 'katakana' (e.g. 加次［蛙]).

(c) The unknown (compound) words are often used.

(d) In some cases, the DS containes wore than one DW.

The extructing procedure has to be constructed with regard to these ploblems.

The relation information are also extracted, that is, 'DH isn't modified' and 'The number of $\mathrm{DW}$ are more than one'.

When DW isn't extracted (that is, DW is neither 'katakana' string nor 'kanji' string nor any entry word in the word dictionary) frow DS, the DS is picked out as check data. (4) Decision of DH-EWl relation

According to the conditions above-mentioned, DH-EW relations are decided.

When extracted relation information is ambiguous, DS is 
picked out as check data.

\section{EXPERIMENTAL RESULTS}

A pilot system has been implemented on FACOM M-360(Nagasaki University Computer Center) and FACOM M-382(Kyushu University Computer Center) mostly by PL/1.

The experimental input data $(2,824 \mathrm{DSs})$ in the first step, are the normalized DSs.

Table 1 shows the number of input, output and check data in each step and the number of correct and incorrect data in output data.

Table 2 shows the extracted DH-EH relations and the number of output data corresponding to the relations.

The experimental results are as follows:

(1) The ratio of IYPE I $(2,374)$ to output data $(2,711)$ is about $87.6 \%$.

(2) The ratin of TYPE II (337) to output data $(2,711)$ is about $12.4 \%$.

(3) The ratio of output data $(2,434)$ to input data $(2,824)$ is about $86 \%$.

(a) The ratio (called system precision) of correct output data $(2,311)$ to output data $(2,434)$ is about $95 \%$.

(b) The ratio(called error ratio) of incorrect output data(123) to output data $(2,434)$ is about $5 \%$.

(4) The ratio of check data(390) to input data $(2,824)$ is about $14 \%$.

Table 1 The Number of Input Data, Output Data and Check Data in Each Step

\begin{tabular}{c|c|c|c}
\hline & $\begin{array}{c}\text { INPUT } \\
\text { DATA }\end{array}$ & $\begin{array}{c}\text { OUTPUT DATA } \\
\text { (correct: incorrect) }\end{array}$ & $\begin{array}{c}\text { CHECK } \\
\text { DATA }\end{array}$ \\
\hline $\begin{array}{c}\text { (1) Extraction } \\
\text { of FE }\end{array}$ & 2,824 & $\begin{array}{c}2,374 \text { (TYPE I ) } \\
337 \text { (TYPE II) }\end{array}$ & 113 \\
\hline $\begin{array}{c}\text { (2) Extraction } \\
\text { of DW }\end{array}$ & 2,711 & $\begin{array}{c}2,502 \\
(2,386: 116)\end{array}$ & 209 \\
\hline $\begin{array}{c}\text { (3) Decision } \\
\text { of Relation }\end{array}$ & 2,386 & $\begin{array}{c}2,318 \\
(2,311:\end{array}$ & $68)$ \\
\hline $\begin{array}{c}\text { Result of } \\
\text { Experiment }\end{array}$ & 2,824 & $\begin{array}{c}2,434 \\
(2,311: 123)\end{array}$ & 390 \\
\hline
\end{tabular}

Table 2 DH-EW Relations and the Number of Output Data corresponding to Each Relation

\begin{tabular}{|c|c|c|c|c|c|c|c|c|}
\hline \multirow{2}{*}{$\begin{array}{l}\text { Relatior } \\
\text { DW : EH }\end{array}$} & \multicolumn{2}{|c|}{ Correct } & \multicolumn{2}{|c|}{ Dubious } & \multicolumn{2}{|c|}{ Incorrect } & \multicolumn{2}{|c|}{ Subtotal } \\
\hline & \multicolumn{2}{|c|}{ Type } & \multicolumn{2}{|c|}{ Type } & \multicolumn{2}{|c|}{ Type } & \multicolumn{2}{|c|}{ Type } \\
\hline & & & & & & & & \\
\hline$\equiv$ & 120 & 103 & 0 & 0 & 0 & 0 & 130 & 103 \\
\hline$<$ & 24 & 11 & 0 & 1 & 0 & 0 & 24 & 12 \\
\hline & 0 & 9 & & 1 & 0 & 1 & 0 & 11 \\
\hline $\mathrm{R}$ & 0 & 10 & 0 & 0 & 0 & 1 & 0 & 11 \\
\hline Subtotal & 2107 & 204 & 3 & 2 & 0 & 2 & 2110 & 208 \\
\hline Total & \multicolumn{2}{|c|}{2311} & \multicolumn{2}{|c|}{5} & \multicolumn{2}{|c|}{2} & \multicolumn{2}{|c|}{2318} \\
\hline
\end{tabular}

Host of incorrect output data occur in the step of extraction of DWs which are described by 'hiragana' notation, because of limitaions of the longest matching method.

The improvement of the results necessitates (a) analysis of the DSs, (b) reinforcewent of the general word dictionary used for extracting the DHs.

\section{CONCLUDING REMARKS}

(1) The similer researches have been carried out about several English dictionarys (e.g. LONGMAN)(2)(3), however there is scaresly any about Japanese dictionary.

(2) We have extracted automaticlly, DW<EH, DWEEH, DW》EW in addition to $\mathrm{DW}>\mathrm{EH}$ as the DW-EW relations.

(3) Input data not suitable for conditions are picked out as check data in each step.

(4) There are a shortage of semantic information (e.g. lack of the adequate DW) in the dictionary because of assuming the human usage of the dictionary.

We have been investigating the followings.

I. Development of a systen for utilizing the dictionary( 7$)$. Il. Development of a syster for hierarchically structuring among entry words in the dictionary( $(i)$.

III. Development of a wan-assisted system for constructing

a practical sized semantic dictionary(4).

\section{ACKNOWLEDGEMENT?}

He will like to thank the member of l'urumaru's laboratory in Nagasaki University, and in paticular, Mr. A.Uchida and Mr. K.Hizuno for their efforts of implementation.

\section{REEERENCES}

(1) S.Yokoyama: Preparation for the Data Management of a Japanese Dictionary, Bul. Electrotech lab., Vol.41, No.11, PP.19-27 (1977.11)

(2) M. Naga o, J.Tujii, Y. Ueda, M.Tai yama: AN ATTEMPT' TO COKPUTERIZED DICTIONARY DATA BASES, PROC. COLING80, PP.534$542(1980.10)$

(3) A.Michiels, J.Noel : APPROACH T0 THESAURUS PRODUCTION, Proc. COLING82, PP. 227-232 (1982.7)

(4) S.Yoshida, H. 'Tsurumaru, "'. Hi taka : MAN- ASSISTED HACHINE CONSTRUCTION OF A SEMANTIC DICTIONARY FOR NATURAL LANGUEGE PBOCESSING, ProC. COLING82, PP. 419-424 (1982.7)

(5) K. Yoshimura, A. Yamasita, T.Hitaka, S. Yoshida : Automatic Extracting System of Technical Terms, NL Technical Report of IPS, 42-1 (1984.3)

(6) H. I'surumaru, K. Mizuno, A.Uchida, T. Hi taka, S. Yoshida: Extraction of Hierarchical Relation between Hords from Definition Sentence of Hord, NL Technical Report of IPS, 45-5 (1984.9)

(7)H.Turumaru, A. Uchida: The Extraction and Organization of Information from the Ordinary Japanese Language Dictionary, Reports of the Faculty Engineering Nagasaki University, Vol.15, Ho.24 (1985.1) 